\title{
II Web 2.0 come mediatore dei processi di apprendimento formali ed informali tra scuola e territorio: il progetto "Didaduezero" della provincia di Trento
}

\section{Corrado Petrucco e Marina De Rossi}

La ricerca presenta una sperimentazione sul campo in collaborazione con l'Istituto Provinciale per la Ricerca e la Sperimentazione Educativa of Trento (IPRASE) e ha sviluppato un nuovo modello (SoSoFIN - Social Software in formal, informal and non-formal context) per verificare se e come i processi di apprendimento degli studenti che si sviluppano nei contesti informali e non formali possono essere con efficacia integrati nel curriculum scolastico formale. Gli insegnanti e gli studenti sono stati accompagnati a sviluppare le loro competenze digitali attraverso l'uso degli strumenti del web 2.0 durante lo svolgimento di specifici progetti disciplinari. La ricerca ha sperimentato un ampio ventaglio di progetti in cui studenti e insegnanti hanno collaborato con le comunità locali creando artefatti digitali fortemente contestualizzati nel territorio e rendendoli disponibili a tutti. Questo approccio didattico, stimolando processi socio-culturali attraverso le tecnologie, ha migliorato la partecipazione e motivazione ad apprendere, così come le competenze digitali di insegnanti e studenti, che sono state messe alla prova sul campo.

\section{Introduzione}

Gli strumenti del Web 2.0 (Wiki, Blog, Social Software, App per mobile, ecc.) sono oggi sempre più utilizzati in molti contesti sociali, da quelli nella vita privata a quelli professionali: è più facile interagire con gli altri, creare artefatti che vengono condivisi on-line e collaborare alla realizzazione di progetti comuni. La recente teoria sul connettivismo di Siemens (Siemens, 2006) sostiene a questo proposito che ora nella Rete non sono importanti solo le informazioni in sé, ma anche e soprattutto chi ne genera i flussi, tanto che diviene sempre più importante essere connessi a una o più reti sociali (Jenkins, 2010). Molte di queste interazioni comunicative hanno luogo negli ambienti informali, ma è ancora raro però che ciò avvenga a scuola. Diversi autori, infatti, sottolineano la forte dicotomia fra le pratiche d'uso delle tecnologie che si fa a scuola e al di fuori di essa, per cui la scuola si presenta loro come un ambiente estraneo rispetto alla 
propria esperienza personale culturale e mediale (McTavish, 2009). È come se gli studenti partecipassero a un doppio curricolo, uno nel formale ed uno nell'informale (tipico della vita quotidiana), quest'ultimo un vero e proprio "curricolo nascosto" (Jenkins, 2006; Ito et al., 2008) e parallelo, che è assorbito appunto informalmente e quotidianamente attraverso le pratiche d'uso delle tecnologie. L'apprendimento in questi contesti si configura come incidentale, auto-diretto e peer-based (Conner, 2009). In questo senso è stata teorizzata una vera e propria learning ecology, (Barron, 2006) per cui diviene importante cercare di capire come si correlano i processi di apprendimento che avvengono a scuola con quelli che si situano all'esterno.

Parte di questi processi esterni alla scuola avvengono on-line con il supporto delle tecnologie e con modalità attive: spesso gli studenti utilizzano la rete per cercare e condividere contenuti come fruitori ma anche per produrne essi stessi, spesso in un'ottica di mash-up, di ri-combinamento dei materiali che risponde a delle specifiche esigenze di un gruppo o di una comunità. I social network in effetti non sono solo fatti di persone, ma di persone e "oggetti» su cui si condividono interessi specifici, per cui possiamo parlare di una vera e propria object-based sociality (Engestrom, 2006). Nell'ottica quindi di una learning ecology che cerchi di considerare anche i processi di apprendimento nell'informale, favorire l'adozione delle tecnologie del Web 2.0 a scuola significa allora ripensare la didattica dei vari contenuti disciplinari facendola entrare in contatto con i contesti informali quotidiani professionali e sociali (Jonassen, 2002) e con i processi dialogici che essi naturalmente sottendono (Cross, 2006). Il contesto didattico entro il quale ci si può muovere può essere quello del project-based learning, (Boss \& Krauss, 2007): ovvero la possibilità di proporre agli studenti dei problemi reali ed autentici che richiedono una soluzione effettiva e che può essere adottata nel contesto sociale, utilizzando soprattutto metodologie collaborative, visto che nella società e nel mondo del lavoro è sempre richiesta la collaborazione di altri per portare a termine con successo un qualsiasi compito che sia professionale, ludico, o legato ad interessi personali

Non è comunque sempre facile utilizzare questo tipo di approcci a causa anche del setting didattico, dei tempi e delle difficoltà di integrazione nel curricolo tradizionale. Inoltre, non aiutano le modalità di comunicazione dei contenuti didattici che nella maggior parte mancano di quella che può essere definita "cornice di realtà" (Castoldi, 2011; Lichtner, 2004). Le modalità di problem solving nella vita quotidiana utilizzano infatti un approccio concreto mentre in molte discipline del curricolo ne viene preferito uno astratto (Resnick, 1995). 


\section{Il modello del progetto: L'integrazione tra apprendimento formale e informale con il Web 2.0}

Proprio sulla base della cornice teorica sopra descritta, è stato pensato il progetto Didaduezero - lo sviluppo delle competenze digitali nella scuola e nel territorio: le opportunità del Web 2.0, che si è svolto nel triennio 2009-2011 in collaborazione con l'Istituto di Ricerca Educativa della Provincia di Trento (IPRASE). Il progetto si poneva come obiettivo l'introduzione dei Social Software e degli strumenti del Web 2.0 a scuola per avviare percorsi didattici inter- e multidisciplinari volti a favorire la partecipazione attiva degli studenti e degli insegnanti nel territorio. I riferimenti pedagogici sono a Dewey, Lewin e Freire, e alla loro interpretazione della scuola come strumento che permette allo studente partecipare in prima persona ai processi di costruzione della conoscenza operando concretamente nella realtà sociale. Perciò, la prima sfida che il progetto ha dovuto affrontare è stata quella di elaborare un modello di innovazione didattica per costruire un ambiente di insegnamento/apprendimento in cui le tecnologie del Web 2.0 aiutassero a fondere assieme gli ambiti formali, non-formali, ed informali (Greenhow et al. 2009; Conole \& Alevizou, 2010; Dabbagh \& Kitsantas, 2012) ove per formale si intendono i processi di apprendimento che avvengono nei contesti della scuola e delle istituzioni formative, per non-formale quelli nei contesti di lavoro o della società, e per informale quelli non legati a luoghi, istituzioni o tempi specifici ma che appartengono comunque all'esperienza quotidiana delle persone.

La seconda riguarda l'obiettivo strategico della costruzione e del governo di reti territoriali, intendendo con quest'ultimo la situazione che si verifica quando determinati attori, individuali e collettivi, provvedono intenzionalmente a progettare, gestire, mantenere un sistema di scambi reciproci. É un modello molto vicino a quello di service learning (Eyler \& Giles, 1999; Butin, 2010; Tapia, 2010). Il service learning è un metodo pedagogico-didattico, che unisce due elementi: il service (volontariato per la comunità) e il learning (l'acquisizione di competenze professionali, metodologiche e sociali). Questo approccio pedagogico che molte scuole in tutto il mondo inseriscono nel proprio curricolo, permette infatti agli studenti di sviluppare le proprie conoscenze e competenze grazie a una pratica di servizio nei confronti della comunità.

Nel caso specifico del nostro progetto Didaduezero tali reti hanno portato alla realizzazione sia di artefatti digitali accessibili su Web condivisi e percepiti come utili da tutta la comunità, sia a vere e proprie azioni comuni nel territorio. Il modello messo a punto (Fig.1) nel corso del progetto, è stato battezzato SoSoFIN (Social Software tra Formale Informale Non formale). 


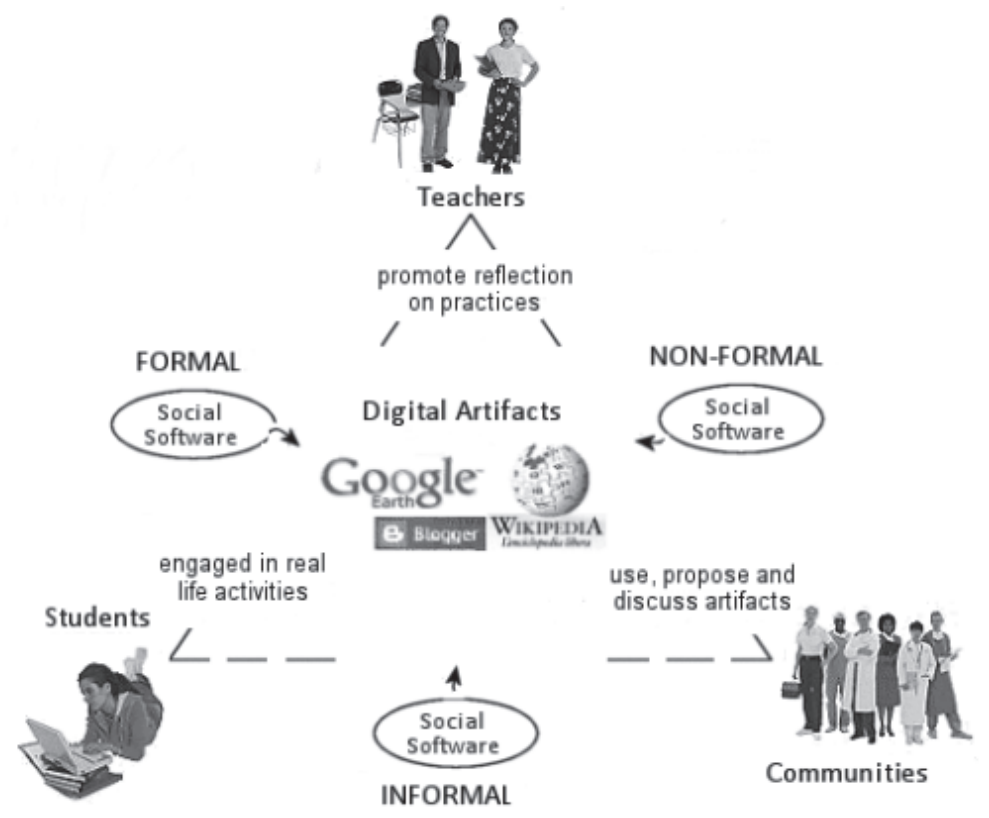

Fig. 1: Il modello SoSoFIN (Social Software tra Formale Informale Non formale) social software possono essere lo strumento per integrare i processi di apprendimento formali, informali e non-formali tra studenti, insegnanti e comunità impegnati a risolvere problemi reali (Petrucco, 2010).

La sfida per gli insegnanti è stata proprio quella di proporre progetti basati su attività di apprendimento concrete che richiedessero l'interazione con comunità on-line e in presenza, anche al di fuori della scuola; comunità capaci di accogliere e di fornire feed-back adeguati per poter generare artefatti digitali condivisi. Il ruolo dell'insegnante diviene quello di facilitatore dei processi di apprendimento contestualizzati e situati nel territorio, stimolando la riflessione sui processi di costruzione degli artefatti digitali.

Qui il concetto di comunità di pratica così come esplicitato da Wenger (2006), diventa un elemento cruciale per gestire il passaggio da una scuola "formale» a una che integra le dimensioni dell'informale e del non-formale, attraverso l'uso dei social software e del project-based learning. Infatti, se la comunità di riferimento dimostrerà di apprezzare gli artefatti realizzati con il contributo degli studenti, la scuola avrà raggiunto due obiettivi fondamentali: formare non solo alla comprensione di contenuti disciplinari, ma anche alla loro contestualizzazione nel sociale. É un modello «rizomatico» (Cormier, 2008; Attwell, 2010) che riscopre una pedagogia del coinvolgimento, come quella proposta da Freire, secondo il quale la comunità può costruire un modello di curricolo ("community as curriculum») non più messo a punto solo da esperti pedagogisti e disciplina- 
risti, ma anche negoziato con le persone che appartengono ai contesti reali di lavoro e di vita.

La sperimentazione del modello, come vedremo, non è stata semplice e le difficoltà incontrate confermano la letteratura sull'argomento (Bull et al. 2008): molti insegnanti hanno rilevato ad esempio che, assieme al fattore limitante del tempo a disposizione, è stata percepita la difficoltà a gestire le attività on-line in classe e a progettare con attenzione quelle on-line da svolgere al di fuori della classe. Inoltre, da parte di tutti gli attori della scuola (insegnanti, genitori e studenti) è spesso emersa una percezione dell'uso dei social software come veri e propri distrattori rispetto alle tradizionali attività scolastiche, misurabili ai fini delle performance (Tan, 2009). Infine è stata confermata una criticità tipica riguardo l'uso delle tecnologie da parte dei docenti: spesso sono praticate a scuola, ma non vissute nel quotidiano, come invece fanno gli studenti. Proprio per questo abbiamo prestato molta cura e attenzione a persuadere gli insegnanti coinvolti a sperimentare le tecnologie anche nel loro ambito informale e non solo a scuola con gli studenti.

\section{Articolazione del progetto, soggetti e obiettivi specifici}

Il progetto ha coinvolto nove scuole della provincia di Trento per un totale di 22 insegnanti (7 della scuola primaria, 11 di secondaria di primo grado e 4 di secondo grado) e circa 300 studenti. Gli obiettivi specifici del progetto consistevano nel verificare se, attraverso un utilizzo pedagogicamente orientato dei Social Software e degli strumenti del Web 2.0, fosse possibile:

1. favorire gli apprendimenti informali e non formali integrandoli in quelli formali;

2. migliorare l'integrazione tra scuola e territorio (istituzioni, enti comunità);

3. aumentare la motivazione ad apprendere creando un ambiente di apprendimento attivo e partecipativo basato su contesti e processi reali;

4. sviluppare le «competenze digitali» di studenti ed insegnanti.

La struttura complessiva del progetto si è articolata nell'arco di tre anni in quattro fasi consecutive:

Promozione e partecipazione. La prima fase del progetto ha visto inizialmente la promozione del progetto stesso nelle scuole. L'obiettivo era orientare gli insegnanti verso una scelta motivata e consapevole in relazione agli obiettivi del progetto.

Orientamento agli strumenti. La seconda fase si poneva l'obiettivo di far comprendere e sperimentare ai docenti, attraverso una serie di incontri laboratoriali, le potenzialità del Web 2.0 e dei Social Software. Durante la seconda fase si 
sono svolti quattro moduli formativi per gli insegnanti su software specifici che suggerivano, nel contempo, anche i potenziali ambiti progettuali che sono stati poi realizzati.

Ricerca-azione nella scuola. La terza fase si è svolta nell'ambito dell'apprendimento formale, in particolare negli spazi del lavoro di classe, e ha visto gli insegnanti elaborare dei progetti didattici all'interno dei vari percorsi disciplinari assieme agli studenti, mettendo alla prova le tecnologie del Web 2.0 scelte, ma senza ancora prevedere esplicitamente la realizzazione di un progetto che coinvolgesse direttamente il sociale e/o il territorio.

Ricerca-azione nei contesti di integrazione scuola-territorio. La quarta fase è stata quella che ha previsto l'apertura e coinvolgimento del sociale e della comunità territoriale d'appartenenza con la realizzazione di progetti specifici attraverso l'uso dei social software e di altri Web 2.0 tools.

\section{I progetti didattici realizzati nei moduli formativi sul Web 2.0}

Al termine dell'attività formativa gli insegnanti hanno elaborato un progetto didattico scelto all'interno degli obiettivi di uno o più moduli scelti tra i seguenti:

Modulo 1: Costruire conoscenza collaborativa:

- Formazione all'uso e alla filosofia di condivisione sociale su cui sono basati i Wiki e Wikipedia, riflessione sulla affidabilità delle fonti; partecipazione come co-autori alla creazione di un testo collaborativo;

- Valorizzazione di risorse locali attraverso Wikipedia;

- Acquisizione di competenze di mash-up di più elementi.

Un progetto, che ha preso come riferimento gli obiettivi di questo modulo, è stato quello realizzato da due insegnanti della scuola secondaria con i loro studenti per la creazione della voce su Wikipedia relativa al Museo etnografico presente nel loro territorio: l'attività ha messo in contatto gli studenti con molti soggetti (studiosi, giornalisti, esperti) e il lavoro di ricerca e inserimento di materiali in Wikipedia è stato riconosciuto positivamente da parte della comunità territoriale, tanto da meritare un articolo sul giornale locale.

Modulo 2: Costruire comunità di lettura:

- Conoscenza della struttura e dei processi relazionali di aNobii e del Blog;

- Utilizzo di tali strumenti in prospettiva di animazione socio-culturale per la formazione del lettore autonomo e critico tra il formale e l'informale (rete tra scuole, biblioteche territoriali e biblioteche virtuali);

- Comprensione dell'importanza dei social software come strumento per la partecipazione attiva alla vita della comunità e diffonderne l'utilizzo come risorsa per la comunità (scuole, musei, risorse del territorio). 
Per quanto riguarda il secondo modulo, un'attività molto efficace è stata quella realizzata da un'insegnante della scuola primaria, che è riuscita a costituire un gruppo di lavoro eterogeneo formato da insegnanti e bibliotecari. Insieme hanno elaborato un progetto avente come sfondo integratore la preistoria. I bambini hanno lavorato su tre diversi percorsi tematici: (a) La preistoria nel racconto fantastico: lettura e recensione racconti fantastici ambientati nella preistoria; (b) La preistoria nei testi scientifici: lettura e recensione di testi di carattere informativo/scientifico adatti alla loro età; e (c) La preistoria nel territorio.

Dopo una visita al locale Museo del Parco Paleontologico di Rovereto e un'analisi del catalogo e di altri materiali documentali, hanno realizzato filmati e foto. Una volta in classe, hanno inventato storie fantastiche sui dinosauri, utilizzando il metodo della scrittura collaborativa con Wiki. Infine, hanno realizzato dei libri digitali, pensati per essere delle vere e proprie «guide per bambini create da bambini» pubblicandoli poi su un blog e su di un'apposita libreria on-line utilizzando il sito della comunità di lettura aNobii. I risultati del progetto sono stati poi presentati alla cittadinanza di Ala in un evento che ha coinvolto genitori, istituzioni e la comunità.

Modulo 3: Condividere risorse geolocalizzate nel territorio:

- Promozione dell'uso dei Social Software di geo-localizzazione, in particolare Google Maps e Google Earth, all'interno di contesti scolastici;

- Sviluppo della capacità di analisi ed interazione della realtà geo-fisica, antropologica e ambientale;

- Sviluppo della capacità di riconoscimento ed integrazione degli artefatti culturali nel contesto territoriale;

- Utilizzo autonomo degli strumenti hardware per la georeferenziazione (GPS, geotagging).

Fra i molti progetti qui realizzati, quello che ha coinvolto maggiormente il territorio è stato quello di alcuni insegnanti della scuola secondaria di primo grado con i loro studenti delle prime e seconde classi: essi hanno individuato una antica strada di comunicazione montana, ora in disuso, ma ricca di riferimenti culturali. Hanno mappato digitalmente il percorso nel territorio grazie all'uso di strumenti GPS e fotografato monumenti e siti d'interesse culturale, con il fine di metterle a disposizione on-line alla cittadinanza, inserendole in una mappa interattiva su Google Earth. Il progetto ha coinvolto molti enti e associazioni della comunità, tra cui l'assessorato alla cultura del Comune, fotoamatori, alpinisti e anche la locale Azienda Sanitaria, interessata a incentivare la comunità del territorio a considerare i vantaggi del benessere fisico che può generare una escursione in montagna seguendo il percorso riscoperto dagli studenti. Ogni attore coinvolto nel progetto infine ha riconosciuto che la scuola aveva offerto veramente qualcosa di utile all'intera comunità. 
Modulo 4: Costruire mappe interculturali del territorio:

- Sviluppo delle competenze di lettura del territorio a livello interculturale e di interazione fra scuola e associazioni locali;

- Promozione di un adeguato utilizzo dei social software che permetta d'organizzare in modo dinamico le informazioni con l'uso di mash-up in particolare con OpenStreetMap

- Avvio di un processo di riflessione sul tema dell'open source nella scuola e nel sociale.

Durante il percorso sono stati aperti un social network su Ning per lo scambio di lavoro e di interessi tra i membri (ragazzi, bibliotecaria, insegnanti) ed un blog per presentare documenti a carattere storico, relazioni ed attività comuni.

\section{Il disegno di ricerca}

Si è fatto riferimento al modello della ricerca partecipante (RP) le cui caratteristiche principali sono: (a) il problema sorge all'interno della comunità che lo definisce, lo analizza e lo risolve; (b) lo scopo è la trasformazione della realtà sociale e la modificazione dei comportamenti di chi ne fa parte; (c) le procedure tendono a stimolare una maggiore consapevolezza nei partecipanti rispetto all'attivazione delle loro risorse; (d) il coinvolgimento dei ricercatori è continuo. La ricerca Didaduezero ha seguito questa prospettiva dando priorità alla costruzione di nuove forme di collaborazione in modo da consentire sia la costante aderenza ai problemi e ai contesti concreti per la verifica sul campo, sia la rigorosità dei procedimenti e dei risultati attraverso l'uso di strumenti adeguati (Bogdan \& Biklen, 1992). La dinamica tra le azioni educativa e scientifica ha coinvolto tutti i partecipanti partendo da ipotesi costruite congiuntamente e centrate sul lavoro degli operatori (Portuois, 1995; Orefice, 2006). Attraverso gli stimoli dei ricercatori, essi sono stati sollecitati a riflettere, a decidere e a predisporre piani d'azione (Trombetta \& Rosiello, 2000).

In sintesi, dal punto di vista metodologico, i tre momenti fondamentali sono stati: (a) la pianificazione del lavoro di gruppo per la progettazione delle azioni riferite ai Moduli; (b) il lavoro partecipato nei vari contesti individuati per la realizzazione dei progetti; e (c) l'indagine che ha accompagnato tutte le fasi dell'azione sul campo per la valutazione in progress (Elliott, 1991). La costruzione del gruppo, per adesione volontaria, è avvenuta mediante incontri-stimolo di presentazione. Insegnanti e i dirigenti scolastici hanno avuto modo di riflettere su alcuni elementi:

- personali rappresentazioni della realtà scuola-territorio,

- concezioni del ruolo e della funzione della scuola nella società,

- percezioni di competenza in relazione alla propria storia professionale,

- percezioni della relazione tra la didattica e l'uso delle tecnologie e sul loro grado di motivazione al cambiamento. 
Si sono poi costituiti sottogruppi per ogni Modulo, in base alle differenti esigenze territoriali; ogni gruppo è stato posto nella condizione di organizzare un repertorio di strategie in ottica di problem posing e problem solving (Kemmis \& McTaggart, 1988). Il progressivo coinvolgimento dei partner territoriali è avvenuto mediante la cooptazione da parte degli insegnanti. In questa fase sono stati effettuati focus group che hanno fatto emergere alcune questioni chiave fondamentali per lo svolgimento del percorso.

Il modello di co-progettazione partecipata tra gli attori coinvolti ha consentito la negoziazione dei bisogni emersi nei vari contesti; infatti, i bisogni e le condizioni erano diverse, in quanto da parte della scuola vi era la necessità di qualificare le risorse del territorio in prospettiva educativa, viceversa da parte delle agenzie del territorio, l'esigenza era poco sentita e l'offerta era limitata. Attraverso le azioni illustrate, incentrate sul continuo scambio-confronto, si sono evidenziate importanti contaminazioni tra i diversi soggetti in una prospettiva di sviluppo di comunità di pratica (Wenger, 2007). La ricerca ha preso in esame la realtà nel suo complesso, considerando le modificazioni delle relazioni e dei comportamenti (Cunningham, 1976), in particolare analizzando la variabile tecnologica. L'intento è stato quello di creare una comunità di ricerca volta a sviluppare un'importante attività critica sull'insieme dei metodi e delle tecniche utilizzati nelle pratiche (Winter, 1987). L'azione si è configurata come uno scambio dialogico continuo tra la competenza scientifica messa in campo dai ricercatori e la competenza pratico-riflessiva degli operatori.

L'obiettivo della ricerca si è evoluto così nella graduale trasformazione spontanea di quelle abitudini e di quei comportamenti che costituivano routine consolidate, ma che non comprendevano alcuna azione di co-progettazione e poca, o nessuna, effettiva integrazione consapevole tra scuola, territorio e comunità virtuali. In effetti, pur essendo diffuso un certo uso delle tecnologie sia a scopo didattico nella scuola (anche a scopo informativo per gli altri partner) l'uso degli strumenti Web 2.0 era pressoché sconosciuto se non come pratica personale a livello informale. Soprattutto non erano mai state considerate le implicazioni processuali di natura comunicativa e collaborativa nella comunità territoriale e virtuale. Sono stati individuati criteri di efficacia (congruenza tra obiettivi educativi e mezzi impiegati nel percorso) e criteri di efficienza (raggiungimento degli obiettivi attraverso l'ottimizzazione delle risorse).

\section{Progettazione condivisa e didattica laboratoriale: \\ due elementi chiave della ricerca-azione}

Due elementi particolarmente significativi nella ricerca-azione sono stati la progettazione condivisa e la didattica laboratoriale.

La progettazione è stata vista come situazione di scoperta che ha privilegiato la conoscenza dei problemi, in contrapposizione con modalità di progettazione lineari; si è adottato un approccio euristico, fortemente orientato all'indagine e fondato su fattori dialogico-discorsivi (Kaneklin, Piccardo \& Scaratti, 
2010). Attraverso l'uso delle tecnologie 2.0, si è voluto evitare il rischio della frammentazione entro contesti di azione separati e autoreferenziali mettendo in atto dispositivi di facilitazione dei processi comunicativi, costruzione di significati condivisi e costruzione di prodotti di scambio. Gli indicatori che hanno consentito il monitoraggio sono stati:

- l'azione vista in funzione all'obiettivo/i del gruppo;

- la centralità delle persone nel ruolo di attori per il cambiamento;

- l'aspetto gestionale del tempo e quello di durata del lavoro in riferimento agli obiettivi;

- il contesto istituzionale, come quadro giuridico-politico entro cui si è svolto il progetto;

- il legame sociale che ha strutturato il processo di animazione socio-culturale sia all'interno del gruppo, sia nel suo rapporto con l'esterno;

- la strategia, quindi il modo per adattare i mezzi agli obiettivi di sviluppo di comunità;

- l'integrazione scuola-territorio.

Il secondo elemento rilevante è stato l'adozione della metodologia laboratoriale che ha rappresentato un fattore in grado di generare forme di apprendimento riflessivo, orientate alla costruzione di conoscenze e abilità nelle quali i soggetti si sono impegnati in un costante dialogo tra la generalità della teoria e la specificità della pratica (Bond \& Walker, 1991). La modalità laboratoriale ha consentito l'emersione dell'implicito, ossia di quei saperi nascosti che nell'agire professionale determinano efficacia nella soluzione di problemi e consapevolezza dei processi sottesi all'esperienza (Polany, 1979; Reber, 1993). Tutte le azioni si sono svolte in questa forma: l'elaborazione dei progetti didattico-educativi riferiti ai quattro Moduli; il coordinamento tra tutti i soggetti coinvolti; le attività svolte direttamente con gli alunni. In tutti i casi, i laboratori si sono configurati come: luogo di integrazione tra la teoria la pratica; spazio di sviluppo di processi cognitivi e riflessivi; contesto metodologico di ricerca «situata», ma capace di far emergere significative generalizzazioni secondo criteri di trasversalità (Gilbert, 2001).

\section{Sintesi dei risultati di ricerca}

Al termine del secondo anno di ricerca sono stati predisposti tre questionari semi-strutturati (domande chiuse e aperte) rivolti ai diversi soggetti appartenenti al contesto scolastico: insegnanti coinvolti direttamente nella ricerca (21), studenti delle classi considerate (228) e insegnanti coinvolti indirettamente (155) al fine di rilevare la percezione, da punti di vista differenti, dei processi d'integrazione degli apprendimenti tra formale e non formale attraverso l'uso dei social software e degli strumenti Web 2.0 (Tabella 1). 
Tabella 1: Sintesi dei questionari somministrati.

\begin{tabular}{|c|c|c|c|}
\hline & TARGET & DIMENSIONI & $\begin{array}{l}\text { TIPOLOGIA E NUMERO } \\
\text { DI DOMANDE }\end{array}$ \\
\hline Q1 & $\begin{array}{l}\text { Docenti } \\
\text { coinvolti } \\
\text { direttamente } \\
\text { nel progetto }\end{array}$ & $\begin{array}{l}1.1 \text { uso personale di internet e social software } \\
\text { (prima e dopo il progetto). } \\
1.2 \text { efficacia di progetti basati su ambienti } \\
\text { educativi integrati sia in ambito pretta- } \\
\text { mente didattico che sul rapporto tra scuola e } \\
\text { comunità. }\end{array}$ & $\begin{array}{l}20 \text { item a risposta chiusa } \\
\text { di cui } 7 \text { richiedevano un } \\
\text { approfondimento in forma } \\
\text { aperta. }\end{array}$ \\
\hline Q2 & $\begin{array}{l}\text { Studenti } \\
\text { partecipanti al } \\
\text { progetto }\end{array}$ & $\begin{array}{l}2.1 \text { gradimento delle attività realizzate. } \\
2.2 \text { percezione sull'efficacia di tale modalità } \\
\text { didattica innovativa. }\end{array}$ & $\begin{array}{l}12 \text { item a risposta chiusa } \\
\text { (scala Likert a } 5 \text { livelli) di } \\
\text { cui } 6 \text { richiedevano una espli- } \\
\text { citazione in forma aperta. }\end{array}$ \\
\hline Q3 & $\begin{array}{l}\text { Docenti } \\
\text { direttamente } \\
\text { o indiret- } \\
\text { tamente } \\
\text { coinvolti nel } \\
\text { progetto }\end{array}$ & $\begin{array}{l}\text { 3.1 appartenenza a un gruppo non-formale o } \\
\text { informale di animazione socio-culturale. } \\
3.2 \text { percezione della relazione tra la propria } \\
\text { appartenenza a un gruppo d'interesse e la } \\
\text { predisposizione all'uso personale e didattico } \\
\text { delle tecnologie. } \\
\text { 3.3 percezione di utilità dell'uso delle tecno- } \\
\text { logie come risorsa per la didattica. }\end{array}$ & $\begin{array}{l}9 \text { item a risposta chiusa di } \\
\text { cui } 6 \text { richiedevano una espli- } \\
\text { citazione in forma aperta. }\end{array}$ \\
\hline
\end{tabular}

Benché gli attori coinvolti nella ricerca-azione appartenessero a molteplici contesti, anche del territorio, gli strumenti di indagine sono stati somministrati esclusivamente a soggetti appartenenti al mondo della scuola poiché si è voluto privilegiare la prospettiva pedagogica volta a costruire ambienti d'apprendimento per una didattica aperta, situata e volta a processi di costante iter-azione, alla base delle quali stanno le pratiche comunicative-sociali che comprendono anche $\mathrm{i}$ social software (Galliani, 2010). Di seguito riporteremo alcuni dei risultati più significativi sui quali basare l'analisi complessiva del progetto; in particolare ci focalizzeremo su alcune dimensioni indagate prima e dopo l'esperienza:

1. L'uso dei Social Software da parte degli insegnanti e le loro percezioni in relazione agli studenti;

2. L'impatto e le ricadute nel territorio dei progetti didattici tra formale e informale mediante utilizzo di Social Software;

3. Il gradimento dimostrato dagli studenti e l'utilità percepita verso le attività del progetto;

4. Le percezioni dei docenti non partecipanti, ma indirettamente coinvolti.

\section{Uso dei Social Software da parte degli insegnanti e le} loro percezioni in relazione agli studenti

Dal quadro delle risposte è emerso che i docenti utilizzavano già abitualmente strumenti del Web sia per uso personale, sia per la preparazione di materiale di lavoro; tuttavia, come è possibile notare dal Grafico 1, la partecipazione al percorso di ricerca-azione ha incrementato percentualmente l'utilizzo di Social Software nella didattica, sia la frequenza d'uso nel contesto informale. I picchi 
maggiori di aumento si sono riscontrati nei seguenti casi: Google Earth (38\%); Google Maps (38\%); Flickr (34\%); aNobii, completamente sconosciuto prima dell'esperienza, (29\%); Skipe (9\%); Blog (19\%). L'aumento complessivo si è in media del 19,5\%. Gli insegnanti hanno poi sottolineato l'influenza decisamente positiva delle tecnologie e dei social software soprattutto perché essi rappresentano una fonte motivazionale per gli studenti e un elemento di novità e d'interesse, in particolare modo se l'attività didattica viene organizzata rispettando i principi dell'apprendimento collaborativo e per scoperta (Grafico 2).

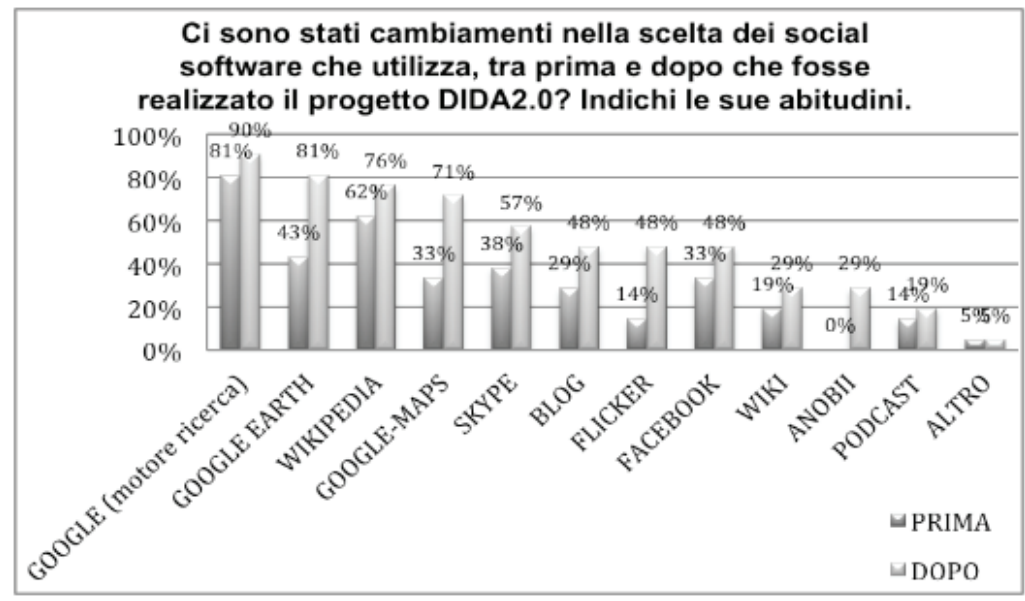

Grafico 1: Percentuali di uso dei differenti social software prima e dopo il progetto.

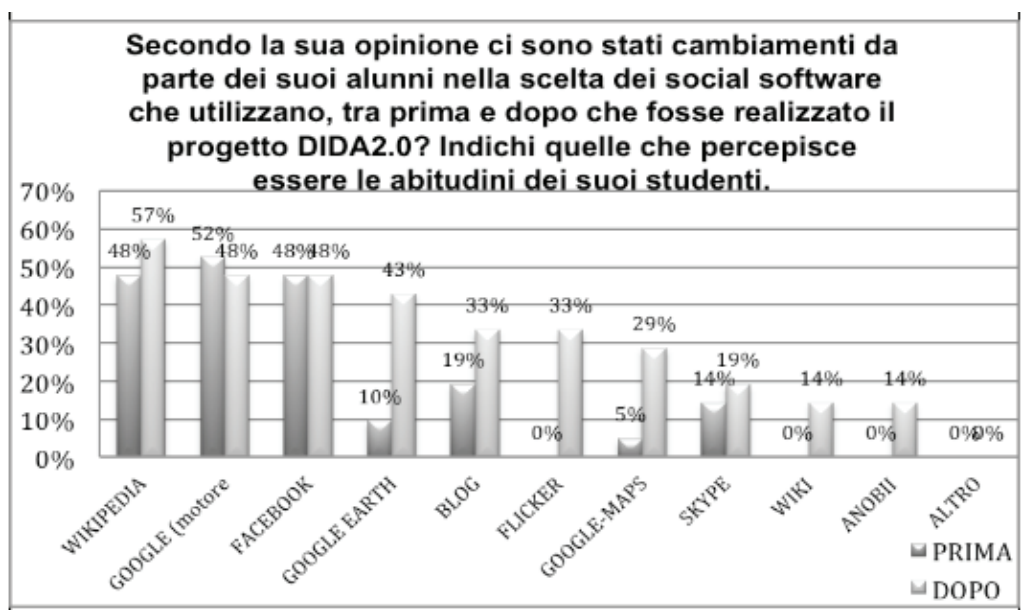

Grafico 2: Percentuali delle risposte degli insegnanti coinvolti nel progetto sulla loro percezione in merito al cambiamento di abitudini dei loro studenti nell'uso dei Social Software in ambito formale e informale. 
Quanto riportato dal Grafico 2 risulta particolarmente interessante andando ad analizzare la tipologia di Social Software: mentre Google motore di ricerca, Facebook e Wikipedia attestano una percezione d'uso aumentata molto poco, gli altri, conosciuti e usati nel progetto, subiscono un incremento notevole (Google Earth 33\%; Flickr 33\%; Google Maps 24\%). Minore aumento in termini di percentuale si riscontra per blog $14 \%$; wiki $14 \%$; aNobii 14\%; tuttavia l'analisi del dato per questi social software si situa nell'ambito degli insegnanti che hanno aderito ai moduli collegati a tali strumenti e la base di partenza, soprattutto per wiki e aNobii era pari a zero. La domanda posta agli insegnanti, seppur non esaustiva in quanto riferita a percezioni, ha avuto la valenza di stimolare la discussione e il confronto in classe tra docenti e alunni sugli usi dei Social Software tra formale e informale. I dati riportati dagli insegnanti vengono confermati nel Grafico 3 che esprime le preferenze degli alunni sui social software e sull'incremento del loro uso, anche per uso scolastico. La serie 1 presenta le risposte date prima della partecipazione al progetto, la serie 2 quelle dopo.

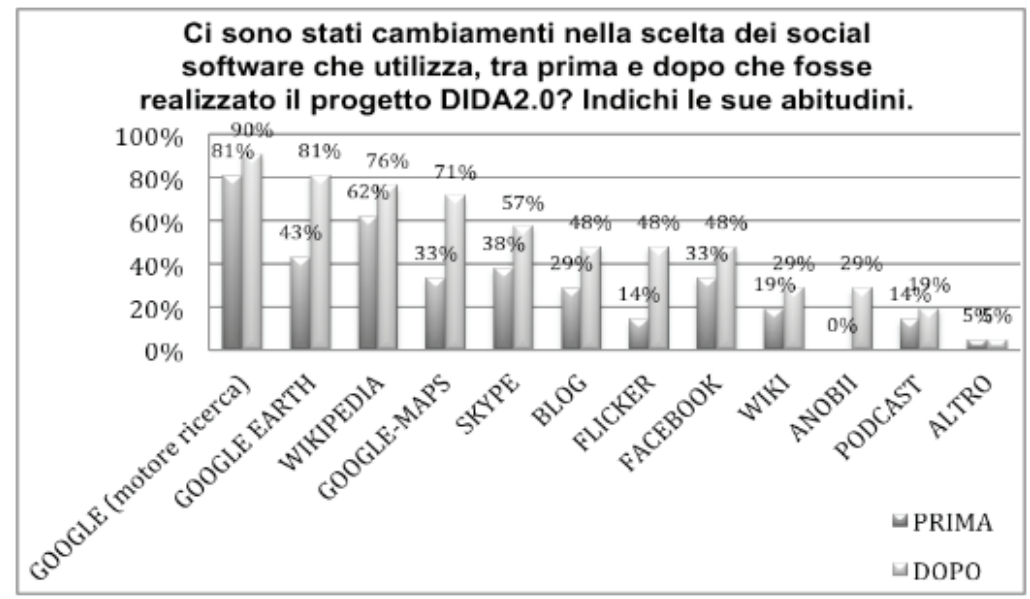

Grafico 3: Risposte degli studenti in relazione alle preferenze e all'uso dei Social Software prima (serie 1) e dopo (serie2) la partecipazione al progetto.

Maggiore dettaglio fornisce la Tabella 2, dalla quale si può evincere quali siano stati i Social Software in cui si è verificato maggior incremento d'uso tra formale e informale.

L'ultima dimensione indagata dal questionario somministrato ai docenti coinvolti nel progetto riguardava le implicazioni del progetto sul rapporto scuola-territorio. Sono da tenere presenti alcuni limiti derivati, in parte, dall'esiguità del gruppo (21 insegnanti), e in parte dalla variabile tempo, in quanto la parte delle attività rivolte al territorio ha avuto una durata piuttosto breve. Tuttavia, la domanda è stata ritenuta necessaria, anche per indagare la disposizione dei docenti a intraprendere percorsi di co-progettazione con enti e agenzie 
del territorio non ideati esclusivamente per le ricadute scolastiche. L'analisi dei dati è riportata con valori assoluti: per quanto riguarda l'uso dei social software per sviluppare progetti scuola-territorio, 13 insegnanti su 21 giudicano abbastanza positivamente l'idea di promuovere con continuità progetti; 4 su 21 molto; 1 moltissimo; 2 poco e 0 per nulla. Complessivamente 18 insegnanti su 21 si attestano su valori positivi.

$\begin{array}{lrrr}\text { Social Software } & \text { \% prima } & \text { \%dopo } & \text { diff. \% } \\ \text { Flickr } & 0,00 \% & 65,00 \% & 65,00 \% \\ \text { Google Maps } & 4,50 \% & 56,30 \% & 51,80 \% \\ \text { Google Earth } & 25,45 \% & 75,40 \% & 49,90 \% \\ \text { Blog } & 34,50 \% & 72,00 \% & 37,50 \% \\ \text { aNobii } & 0,00 \% & 35,00 \% & 35,00 \% \\ \text { Wiki } & 0,00 \% & 32,00 \% & 32,00 \% \\ \text { Skype } & 15,20 \% & 35,00 \% & 19,80 \%\end{array}$

Tabella 2: Social Software che hanno avuto maggior aumento d'uso da parte degli studenti in percentuale.

\section{Gradimento delle attività e percezione di efficacia degli studenti}

Il questionario rivolto agli studenti è stato inviato agli insegnanti referenti che hanno provveduto a somministrarlo seguendo il protocollo costruito dal gruppo di ricerca universitario. Complessivamente sono stati coinvolti 228 studenti di cui il $52 \%$ maschi e il $48 \%$ femmine, variamente ripartiti a seconda del grado scolastico: $20 \%$ scuola primaria; $72 \%$ scuola secondaria di I grado e $8 \%$ secondaria di II grado. Dai dati ottenuti è emerso che la maggior parte degli studenti abbia apprezzato l'uso dei social software e degli strumenti Web 2.0 nella didattica in prospettiva d'uso tra formale e informale (Grafico 4).

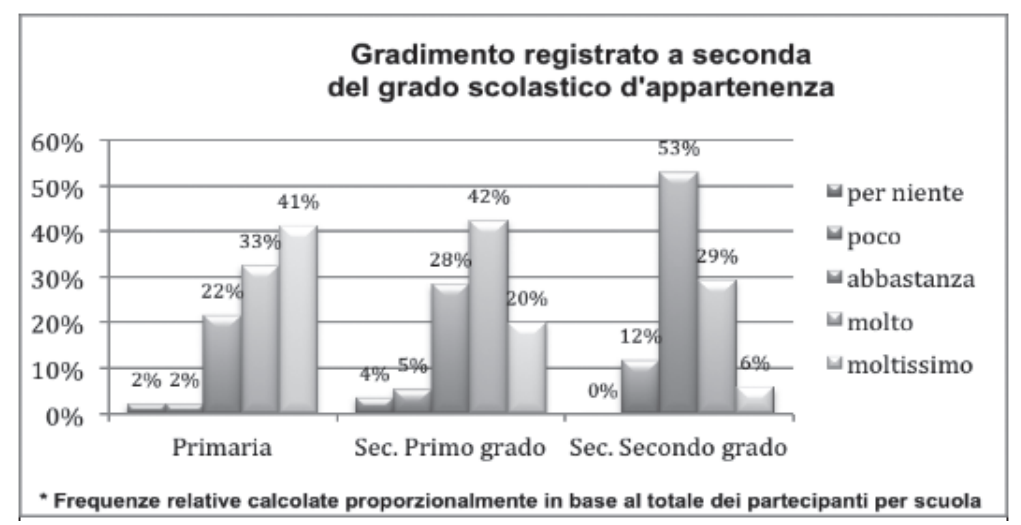

Grafico 4: percentuali del gradimento espresso dagli studenti sulle attività proposte dal progetto. 
In tal senso non si riscontrano differenze determinate dal genere, ma a seconda del modulo e dell'ordine scolastico d'appartenenza. Mentre i più piccoli hanno apprezzato il progetto, accogliendolo con entusiasmo e interesse, man mano che aumenta il livello di scolarizzazione e quindi l'età anagrafica dei soggetti, il giudizio si diversifica e cresce la percezione dell'utilità rispetto a quella del semplice gradimento. In conclusione sembra che l'introduzione degli strumenti Web 2.0 abbia avuto un impatto positivo principalmente in tre ambiti:

1. Didattica (Grafico 5);

2. Collaborazione e instaurarsi di relazioni significative con insegnanti e compagni;

3. Instaurarsi di relazioni e rapporti con il territorio e soggetti esterni (Grafico 6).

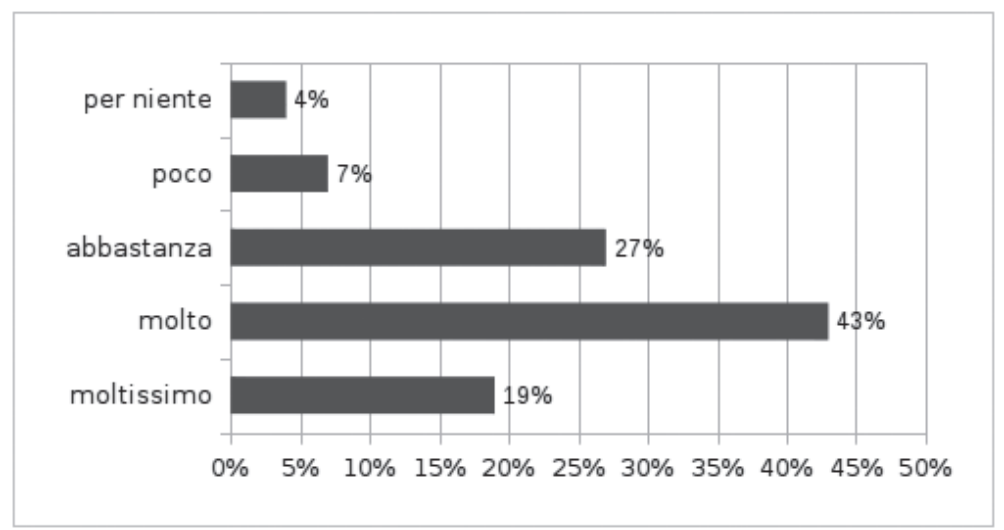

Grafico 5: Percezione di utilità delle attività nella didattica.

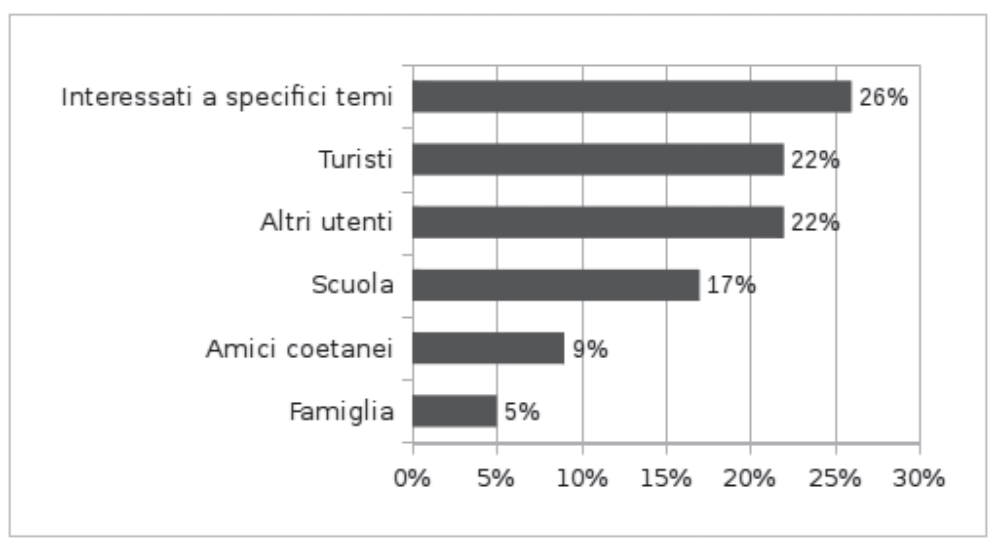

Grafico 6: Percezione di utilità delle attività sviluppate nei rapporti con l'esterno. 
A proposito delle risposte indicate dal Grafico 6 è interessante notare che gli studenti, pur avendo svolto le attività proposte dal progetto prevalentemente a scuola e sviluppate come lavoro fortemente connesso alla didattica curricolare, hanno indicato ricadute e destinatari di contesti extrascolastici. Sollecitati a spiegare i dati emersi dalle loro risposte, la maggioranza ha dichiarato di aver pensato a soggetti non solo coetanei, ma a una condivisione allargata derivata dalla progressiva consapevolezza acquisita durante il percorso che attraverso gli strumenti Web 2.0 le potenzialità comunicative si ampliano a dismisura.

\section{Percezione dei docenti sulle pratiche didattiche con gli strumenti del Web 2.0}

Il terzo e ultimo strumento di rilevazione, somministrato ai docenti direttamente ed indirettamente coinvolti nel progetto (complessivamente 155), ha messo in evidenza due importanti questioni: da un lato che la presenza di un collega esperto di tecnologie resta una variabile discriminante per l'introduzione di pratiche didattiche innovative, poiché in grado di fronteggiare gli eventuali imprevisti che potrebbero presentarsi nel corso della lezione e suggerire possibile utilizzi (61\% molto; $21 \%$ moltissimo). Dall'altro, la necessità di organizzare gruppi di lavoro tra insegnanti della medesima disciplina (e solo in un secondo tempo interdisciplinari) e tecnici esperti per co-progettare attività didattiche equilibrate sia dal punto di vista tecnologico che contenutistico $(56 \%$ molto; $17 \%$ moltissimo). Un aspetto particolarmente interessante, riportato nel Grafico 7 , è emerso dalla domanda in cui gli insegnanti hanno spiegato quali erano secondo loro le strategie didattiche più efficaci per l'integrazione dell'uso dei social software nella didattica.

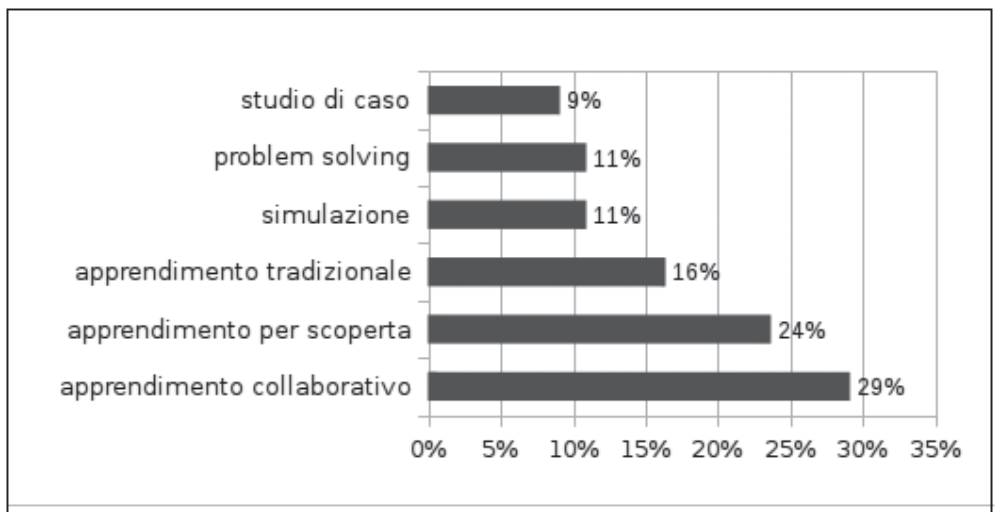

Grafico 7: Percezione della tipologia di strategie didattiche più idonee a favorire l'integrazione dei social software 
La maggior parte delle strategie sono riconducibili un approccio metodologico attivo e per competenze; tuttavia, dalle riflessioni aperte che erano consentite collegate alla domanda, anche le modalità didattiche tradizionali sono state viste come possibile scenario per l'integrazione delle tecnologie 2.0 nella didattica grazie ad una gestione competente dell'insegnante. La poca preparazione dei docenti è stata vista come un effettivo ostacolo e deterrente all'innovazione più di quanto lo siano le carenze strumentali e organizzative, tempi scolastici compresi.

\section{La rilevazione delle concezioni sul rapporto scuola-territorio: focus group e video interviste}

Il gruppo di ricerca ha cercato di rilevare mediante 6 focus group le principali concezioni sui rapporti scuola-territorio e gli strumenti del Web 2.0, nelle comunità di riferimento. In questo modo si è potuto anche co-progettare l'intervento di ricerca-azione, seguendo le specifiche peculiarità territoriali. I 48 partecipanti dei focus group provenivano dal contesto formale (insegnanti, dirigenti), non-formale (enti comunali, biblioteche, musei, centri di documentazione), informale (agenzie educative/animative, associazioni culturali e interculturali, cooperative di promozione turistica, associazioni di categoria). L'analisi testuale delle diverse discussioni (mediante il software Atlas.ti) ha seguito sia un approccio top-down relativo alle dimensioni che si intendevano indagare, sia uno bottom-up riferito soprattutto alla fase progettuale dei lavori. Si sono individuate 6 specifiche "famiglie» in cui si sono aggregati gli 85 codici relativi alle concezioni inerenti la scuola ( 12 codici), il territorio ( 15 codici), le nuove tecnologie informatiche $(15$ codici), le finalità del progetto (16 codici), le modalità realizzative del progetto (10 codici), le difficoltà del progetto (17 codici).

Entrando nello specifico delle principali dimensioni indagate, emerge come la scuola non sia concepita unicamente come agenzia di formazione. Essa ha un ruolo fondamentale per la comunità come collante del territorio, come produttrice di conoscenza e come entità in grado di promuovere il territorio stesso. Tuttavia, nonostante l'apparente apertura di questa istituzione nei confronti del territorio, non sempre è stato possibile instaurare dei rapporti costruttivi con gli enti territoriali. Questo aspetto dipende sia da una certa rigidità nella struttura scolastica e nella sua burocrazia, che da una mancanza di tempo ed energia dei docenti stessi. I soggetti coinvolti individuano 4 aspetti principali del territorio da valorizzare:

- la cultura (intesa come tradizioni),

- l'economia (prodotti locali e turismo),

- l'ambiente (o meglio la natura) e

- le relazioni interpersonali di carattere solidaristico. 
I focus group evidenziano una visione che associa le nuove tecnologie informatiche (internet in particolare) alla semplice possibilità di ottenere pubblicitàl visibilità a basso costo. Non sempre la rete è intesa come ambiente di scambio e di co-costruzione della conoscenza. Spesso i soggetti, sia del mondo della scuola che dei contesti non formali e informali, esprimono diffidenze nei confronti di Internet, che viene percepito come un mezzo futile, che distrae i bambini e che non favorisce una rielaborazione critica dei contenuti. I partecipanti sono comunque convinti che l'utilizzo delle tecnologie dell'informazione e comunicazione in progetti didattici, in cui gli studenti sono protagonisti, aumenti la motivazione dei bambini e permetta di sperimentare con successo nuove modalità di fare lezioni trasversali alle diverse discipline scolastiche. Le finalità del progetto, espresse nei focus group, sono riconducibili a due interessi, apparentemente distinti, ma in realtà fortemente interdipendenti: da una parte, ci sono le esigenze educative di fornire nuove competenze digitali agli studenti e di aumentare la loro conoscenza del territorio; dall'altra, la necessità di promuovere il territorio stesso e di incrementare le reti sociali.

L'obiettivo di rendere i ragazzi produttori attivi di conoscenza mediante l'uso dei Social Software si coniuga quindi con il rafforzamento dell'identità territoriale. I ragazzi, mediante gli strumenti Web 2.0, divengono così l'anello di collegamento tra scuola, famiglia, territorio. Divengono fondamentali le definizioni di computer e software, non come oggetti di studio, ma come strumenti applicativi. L'approccio didattico deve prevedere il protagonismo degli studenti, il lavoro collaborativo e la visibilità pubblica degli artefatti realizzati a scuola anche se viene riconosciuto che la progettazione e la produzione necessitano di tempi lunghi e dilatati. Sia secondo gli insegnanti, sia secondo gli attori altri, vi è la convinzione che gli enti territoriali e la scuola devono interagire in modo sinergico e concreto, sia mediante incontri in presenza che attraverso attività on-line. Il progetto Didaduezero è stato anche terreno di riflessione e scambio sulle criticità del lavoro progettuale in quanto si sono riscontrate diverse difficoltà e resistenze in merito. Si è evidenziato come spesso vi sia una mancanza di collaborazione e scambio: tra colleghi a scuola (causata anche al divario di competenze informatiche e digitali); tra scuola, enti e altre agenzie del territorio; tra la scuola e le famiglie degli studenti. Questo, di fatto, non favorisce processi costanti di partecipazione attiva e co-progettazione, se non in occasioni sporadiche e sostenute da attivatori esterni, come nel caso in oggetto.

Per quanto riguarda il territorio si è sottolineata la mancanza di tempo da parte dei diversi attori sociali, il gap intergenerazionale (culturale e tecnologico) e la carenza di strumenti tecnologici performanti a disposizione della comunità e della scuola. Tuttavia, complessivamente è stato valutato positivamente l'impatto e la ricaduta dell'esperienza per quanto riguarda un cambiamento di atteggiamento nei confronti delle TIC introdotte tra scuola e territorio, come si può notare dallo schema che sintetizza alcune delle concezioni emerse durante i focus group. 
Nella Didattica

$$
\begin{gathered}
\text {-approccio trasversale } \\
\text {-facilitazione relazionale } \\
\text {-aumento informazioni } \\
\text {-connessione tra apprendimento formale e } \\
\text { informale }
\end{gathered}
$$

Tra scuola e territorio

-risorsa informativa

abbattimento distanza

-vetrina, pubblicità

-abbattimento dei costi

-scambio continuo

-comunicazione istituzionale

-risorsa (turismo)
Studenti/Insegnanti

-aumento motivazione per gli studenti

-coinvolgimento attivo

-più risorse per il lavoro degli insegnanti

Tabella 3: Schema di sintesi di alcune concezioni emerse dai diversi soggetti all'interno dei focus group relative alla Tecnologia

\section{Conclusioni e sintesi dei risultati}

In sintesi, dall'analisi dei dati rilevati attraverso i diversi strumenti, è possibile sostenere che l'uso del Web 2.0 secondo il modello proposto e nel contesto sperimentale indagato ha soprattutto:

- Favorito nella scuola l'integrazione tra conoscenze formali ed informali;

- Favorito processi di co-progettazione e animazione socio-culturale nella comunità territoriale;

- Facilitato l' apprendimento basato su problemi reali;

- Caratterizzato la centralità dell'apprendimento rispetto alla centralità dell'insegnamento;

- Aumentato la partecipazione e la motivazione degli alunni;

- Aumentato le risorse a disposizione degli insegnanti.

Tra gli aspetti più rilevanti emersi a fine progetto, risulta come i social software, se utilizzati in maniera consapevole, finalizzata e ragionata favoriscano l'integrazione tra apprendimento formale ed informale ed avvicinino i ragazzi alle potenzialità del web rendendoli effettivamente produttori di risorse utili per l'intera comunità. In particolare si constata, da un lato, l'aumento di motivazione e di coinvolgimento dei minori (anche di coloro che solitamente hanno difficoltà relazionali e cognitive) nelle attività didattiche; dall'altro, si evidenza come gli studenti si sentano veramente protagonisti di quello che fanno e quindi maggiormente responsabilizzati da questi progetti. Il processo di apprendimento viene così reso più significativo.

Sembra quindi che l'utilizzo del Web 2.0 a fini didattici non migliori le competenze digitali degli alunni, ma attivi anche nuove forme di intelligenza 
creativa e di collaborazione sia tra pari che tra docenti e studenti. Grazie ai prodotti realizzati nel progetto Didaduezero si è poi stretto un maggiore contatto tra scuola e territorio, sia in termini di conoscenze delle sue caratteristiche, sia in termini di ricadute positive per le comunità stesse. Queste si sono arricchite di nuove risorse multimediali (realizzate e condivise on line dai ragazzi) fruibili da tutti e in grado di valorizzare ulteriormente il territorio e le sue reti sociali.

$\mathrm{Si}$ deve sottolineare infine un risultato importante che si è ottenuto in questo progetto: il coinvolgimento delle famiglie degli studenti. I genitori hanno infatti supportato il lavoro scolastico, agevolando la scoperta delle ricchezze del posto, e hanno potuto monitorare passo dopo passo, semplicemente collegandosi on line, le attività svolte dai propri figli.

I lavori delle diverse scuole e la documentazione dell'intero processo di attuazione del progetto (con contributi testuali e con le video interviste realizzate) sono stati raccolti in un apposito spazio web aperto a tutti: http://www.iprase. tn.it/didaduezero/index.php

\section{Nota degli autori}

Il contributo è suddiviso nel seguente modo (titoli dei paragrafi abbreviati):

Corrado Petrucco: Introduzione; Il modello del progetto; Articolazione del progetto; Conclusioni.

Marina De Rossi: Introduzione; Il disegno della ricerca; Sintesi dei risultati; La rilevazioni delle concezioni.

\section{Riferimenti Bibliografici}

Anderson, T. (2008). The theory and practice of online learning (2nd ed.). Athabasca University (AU) Press, Edmonton, AB.

Atwell, G. (2010). Can Web 2.0 and Social Software Help Transform How We Measure Quality, in Teaching, Learning, and Research? Changing Cultures in Higher Education, Volume I. Springer-Verlag Berlin Heidelberg, p. 433.

Barron, B. (2006). Interest and self-sustained learning as catalysts of development: A learning ecologies perspective. Human Development, 49, 193-224.

Bogdan, R. C., \& Biklen, S. K. (1992). Qualitative Research for Education. Boston: Allyn and Bacon.

Bond, D., \& Walker, D. (1991). Experience and Learning: Reflection at Work. Gelong: Deakin University Press.

Boss, S., \& Krauss, J. (2007). Reinventing project-based learning: Your field guide to real-world projects in the digital age. Eugene, OR: International Society for Technology in Education.

Bull, G., Thompson, A., Searson, M., Garofalo, J., Park, J., Young, C., \& Lee, J. (2008). Connecting informal and formal learning: Experiences in the age of participatory media. Contemporary Issues in Technology and Teacher Education, 8(2). Available online at: http:// www.citejournal.org/vol8/iss2/editorial/article1.cfm

Butin, D. W. (2010). Service-Learning in Theory and Practice: The Future of Community Engagement in Higher Education. New York: Palgrave Macmillan.

Castoldi, M. (2011). Progettare per competenze. Percorsi e strumenti. Roma: Carocci.

Conole, G., \& Alevizou, P. (2010). A literature review of the use of Web 2.0 tools. Higher Education Report, Milton Keynes: Open University. 
Cormier, D. (2008). Rhizomatic education: community as curriculum. Innovate 4(5). Retrived online on October 20th, 2014, from http://www.innovateonline.info/index. php?view=article \&id $=550$

Dabbagh, N., \& Kitsantas, A. (2012). Personal Learning Environments, social media, and self-regulated learning: A natural formula for connecting formal and informal learning. The Internet and Higher Education, 15(1), 3-8.

Eyler, J., \& Giles Jr., D. E. (1999). Where's the Learning in Service-Learning? San Francisco: Jossey-Bass.

Elliott, J. (1991). Action Research for Educational Change. Buckingham: Open University Press.

Ferri, P. (2011). Nativi Digitali. Milano: Mondadori.

Gilbert, K. R. (2001). The emotional nature of qualitative research. B. Raton: CRC Press.

Greenhow, C., Robelia, B., \& Hughes, J. (2009). Web 2.0 and classroom research: What path should we take now? Educational Researcher, 38(44), 246-259.

Ito, M., Horst, H. A., Bittanti, M., Boyd, D., Herr-Stephenson, B., Lange, P. G., Pascoe, C.J. \& Robinson, L. (2008). Hanging Out, Messing Around, Geeking Out: Living and Learning with New Media. Chicago: MacArthur Foundation. Available online at: http://digitalyouth. ischool.berkeley.edu/report

Jenkins, H. (2010). Culture partecipative e competenze digitali. Milano: Guerini.

Jenkins, H., Clinton, K., Purushotma, P., Robinson, A. J., \& Weigel, M. (2006). Confronting the Challenges of Participatory Culture: Media Education for the 21st Century. Chicago: MacArthur Foundation. Available on line at http://www.digitallearning.macfound.org/

Kaneklin, C., Piccardo, C., \& Scaratti, G. (2010). La ricerca-azione: cambiare per conoscere nei contesti organizzativi. Milano: Cortina.

Lichtner, M. (2004). Valutare l'apprendimento. Teorie e metodi. Milano: FrancoAngeli.

McTavish, M. (2009). I get my facts from the Internet: A case study of the teaching and learning of information literacy in in-school and out-of-school contexts. Journal of Early Childhood Literacy, 9(1), 3-28.

Orefice, P. (2006). La Ricerca Azione Partecipativa: teorie e pratiche. Napoli: Liguori.

Pourtois, J. P. (1995). La ricerca-azione in pedagogia. In E. Becchi, B. Vertecchi (a cura di), Manuale critico di sperimentazione e della ricerca educativa, Milano: FrancoAngeli.

Reber, A. S. (1993). Implicit Learning and Tacit Knowledge: An Essay on Cognitive Unconcius. New York: Oxford University Press.

Siemens, G. (2006). Knowing Knowledge. Retrived on October 20th, 2014, from www. knowingknowledge.com

Tan, J. P-L. (2009). Digital kids, analogue students : a mixed methods study of students' engagement with a school-based Web 2.0 learning innovation. Unpublished dissertation. Retrived on October 20th, 2014 from http://eprints.qut.edu.au/30396/

Tapia, M. N. (2010). Service Learning Widespread in Latin America. Phi Delta Kappan 91(5), 31-32.

Wenger, E. (2007). Comunità di pratica. Milano: Cortina.

Parole chiave: Web 2.0, social software, scuola, apprendimento formale, apprendimento informale, comunità territoriali. 


\section{Das Web 2.0 als Mediator zwischen formalen und non- formalen Lernprozessen zwischen Schule und Gemeinschaft: Das Projekt "Didaduezero" in der Provinz Trient}

\section{Zusammenfassung}

Im vorliegenden Projekt wurden in Zusammenarbeit mit dem Istituto Provinciale per la Ricerca e la Sperimentazione Educativa of Trento-Italy (IPRASE) ein neues Versuchsmodell (SoSoFIN - Social Software in formal, informal and non-formal context) entwickelt, um zu untersuchen wie Lernprozesse im Web 2.0 in informellen und nicht-formalen Kontexten gewinnbringend in Lehrpläne integriert werden können. Lehrpersonen, Schülerinnen und Schüler wurden darin unterstützt, Kompetenzen im Umgang mit diese Werkzeugen in schulischen Projektarbeiten zu entwickeln. Abgedeckt wurde ein breiter Bereich von Web 2.0-Projekten, in denen Schülerinnen und Schüler sowie Lehrpersonen mit lokalen Gemeinden digitale Erzeugnisse kreierten und sie allen zur Verfügung stellten. Dieser Zugang stimulierte sozial-kulturelle Prozesse, erhöhte die Lernmotivation und -beteiligung ebenso wie die digitalen Fertigkeiten der Schülerinnen, Schüler und Lehrpersonen, was Tests im Feld zeigten.

Schlagworte: Web 2.0, social software, Schulische Vermittlung, formales Lernen, informelles Lernen, lokale Gemeinden

\section{Le Web 2.0 comme médiateur des processus d'apprentissage formels et informels entre l'école et la société: Le projet "Didaduezero» dans la province du Trient}

\section{Ré sumé}

Le projet, mené en collaboration avec l'Istituto Provinciale per la Ricerca e la Sperimentazione Educative de Trento (IPRASE)-en Italie, a développé un nouveau modèle de recherche (SoSoFIN) visant à expérimenter comment les processus d'apprentissage des étudiants en contexte informel et non formel peuvent être médiatisés et intégrés avec succès dans le curriculum scolaire formel. Les enseignants et les étudiants ont été soutenus dans le développement de compétences digitales en utilisant le Web 2.0 dans le cadre de projets disciplinaires spécifiques. La recherche couvre un large éventail de projets d'apprentissage lors desquels les élèves et les enseignants collaborent à l'aide du Web 2.0 avec la communauté locale en créant des artefacts digitaux hautement contextualisés dans le monde social et en les rendant accessibles à tous. Les résultats de la recherche montrent que cette approche stimule, grâce au recours à la technologie, les processus socioculturels, renforce la participation et la motivation à apprendre des étudiants tout en améliorant leurs compétences digitales ainsi que celles des enseignants.

Mots-clés: Web 2.0, social software, médiation scolaire, apprentissage formel, apprentissage informel, communautés locales. 


\section{The Web 2.0 to mediate formal and informal learning processes between school and social community: The "Didaduezero" project in the Provincia of Trento}

\section{Summary}

The research, carried out in collaboration with the Istituto Provinciale per la Ricerca e la Sperimentazione Educativa of Trento-Italy (IPRASE), developed a new experimental model (SoSoFIN - Social Software in formal, informal and non-formal context) to investigate how learning processes in formal and informal contexts can be mediated and successfully integrated within the formal school curriculum. Teachers and students were supported to develop their digital competences using these tools at school during their project based learning activities. The research covers a wide range of Web 2.0 learning projects during which students and teachers collaborated with local communities creating highly contextualized digital artefacts, and making them available to all. Results show that this approach stimulates social-cultural processes, increases students participation and motivation to learn, as well as digital skills of both students and teachers.

Keywords: Web 2.0, social software, school mediation, formal learning, informal learning, local community. 
